Research Paper

\title{
Circulating and Pulmonary T-cell Populations Driving the Immune Response in Non-HIV Immunocompromised Patients with Pneumocystis jirovecii Pneumonia
}

\author{
Nan-Nan Zhang1,2,3,4, Xu Huang1,2,3, Hui-Ying Feng1,2,3,4, Lin-Na Huang1,2,3, Jin-Gen Xia 1,2,3, Yan Wang4, Yi \\ Zhang ${ }^{1,2,3}$, Xiao-Jing $\mathrm{Wu}^{1,2,3}$, Min Li 1,2,3, Wei Cui ${ }^{5}$, and Qing-Yuan Zhan ${ }^{1,2,3}$ \\ 1. Center for Respiratory Diseases, China-Japan Friendship Hospital, Beijing 100029, China \\ 2. Department of Pulmonary and Critical Care Medicine, China-Japan Friendship Hospital, Beijing 100029, China \\ 3. National Clinical Research Center for Respiratory Diseases, Beijing 100029, China \\ 4. Graduate School of Peking Union Medical College, Chinese Academy of Medical Sciences, Beijing 100730, China \\ 5. Beijing Key Laboratory of Remodeling-Related Cardiovascular Diseases, Beijing Anzhen Hospital of Capital Medical University, Beijing Institute of Heart \\ Lung and Blood Vessel Diseases, Beijing 100029, China. \\ $\square$ Corresponding author: Qing-Yuan Zhan, Center for Respiratory Diseases, China-Japan Friendship Hospital; Department of Pulmonary and Critical Care \\ Medicine, China-Japan Friendship Hospital; National Clinical Research Center for Respiratory Diseases; Graduate School of Peking Union Medical College, \\ Chinese Academy of Medical Sciences, Beijing, China. No 2, East Yinghua Road, Chaoyang District, Beijing 100029, China. Tel.: +86-218-420-6119; Fax.: \\ +86-218-420-5110; Email: doctormtxyy@163.com
}

(1) The author(s). This is an open access article distributed under the terms of the Creative Commons Attribution License (https://creativecommons.org/licenses/by/4.0/). See http://ivyspring.com/terms for full terms and conditions.

Received: 2019.03.01; Accepted: 2019.07.17; Published: 2019.08.14

\begin{abstract}
Background: Previous studies in human subjects have mostly been confined to peripheral blood lymphocytes for Pneumocystis infection. We here aimed to compare circulating and pulmonary T-cell populations derived from human immunodeficiency virus (HIV)-uninfected immunocompromised patients with Pneumocystis jirovecii pneumonia (PCP) in order to direct new therapies.

Methods: Peripheral blood and bronchoalveolar lavage samples were collected from patients with and without PCP. Populations of Th1/Tcl, Th2/Tc2, Th9/Tc9, and Th17/Tc17 CD4 ${ }^{+}$and CD8 ${ }^{+} \mathrm{T}$ cells were quantified using multiparameter flow cytometry.

Results: No significant differences were found between PCP and non-PCP groups in circulating $T$ cells. However, significantly higher proportions of pulmonary Thl and Tc9 were observed in the PCP than in the non-PCP group. Interestingly, our data indicated that pulmonary Thl was negatively correlated with disease severity, whereas pulmonary Tc9 displayed a positive correlation in PCP patients.

Conclusions: Our findings suggest that pulmonary expansion of Th1 and Tc9 subsets may play protective and detrimental roles in PCP patients, respectively. Thus, these specific T-cell subsets in the lungs may serve as targeted immunotherapies for patients with PCP.
\end{abstract}

Key words: Pneumocystis jirovecii pneumonia, HIV negative, immunocompromised, T cells

\section{Introduction}

Pneumocystis jirovecii pneumonia (PCP) is a serious infectious disease currently afflicting immunocompromised populations [1]. Although the incidence of PCP in human immunodeficiency virus (HIV)-positive patients has been reduced as a consequence of improved access to effective antiretroviral therapy, the risk has increased among patients who are immunocompromised due to other causes $[2,3]$. For example, patients with autoimmune diseases, or who are receiving cancer chemotherapy or organ transplants are highly susceptible to severe PCP [4]. The mortality rate in non-HIV patients with 
PCP ranges between 30 and 50\%, which is significantly higher than the 7 to $17 \%$ seen in HIV-positive populations [5]. An emerging concern is that increasing numbers of non-HIV patients with PCP are being admitted to intensive care units (ICU). Among this population, mortality rates have reached $58 \%$ to $80 \%$ [6-8].

There is a growing body of evidence that recognizes the critical role of the host immune response to $P$. jirovecii $[9,10]$. The essential role of $\mathrm{CD}^{+} \mathrm{T}$ cells in defense against $P$. jirovecii in animal models and patients is well documented [11, 12]. However, $\mathrm{CD}^{+} \mathrm{T}$ cell mediated immunity may also be pathogenic, resulting in substantial lung damage during $P$. jirovecii infection by increasing inflammatory mediators, and recruiting effector cells, particularly in the process of immune reconstitution [13-15]. Specific subsets of $\mathrm{CD}^{+} \mathrm{T}$ cells have been reported to mediate the immune response in animals with $P$. murina infection. In mice inoculated with $P$. carinii, Th1 and Th2 subsets were recruited into the lungs, with the response mediated predominantly by Th2 cells [16]. Mice deficient in either IFN- $\gamma$ or IL-4 were still able to resolve their infections [17, 18]. Additionally, the IL-17/IL-23 axis was capable of extending the Th17 effector response [19], and Th17 cells are involved in the immune response during $P$. jirovecii infection [20]. More recently, IL-9-\% mice showed reduced $P$. murina burden in the lungs [21]. The presence of $\mathrm{CD}^{+} \mathrm{T}$ cells in response to $P$. jirovecii infection may also be deleterious [22]. Of interest, it has been shown that high level Tc1-mediated production of IFN- $\gamma$ could confer a protective effect that Tc2 cells do not in P. murina-infected animals [23, 24].

This body of research draws our attention to roles of $\mathrm{T}$ cells in $P$. jirovecii infection. Therefore, a better understanding of the Pneumocystis-related immune response might come from focusing on distinct T-cell subsets. To date, apart from a case-control study that described no significant differences in circulating $\mathrm{T}$ cells, including Th1, Th2, and Th17 subsets between renal transplant patients with and without PCP, studies of T-cell subset mediated immunity in PCP patients remain sparse [25]. Studies of T-cell immunity in human subjects have been mostly restricted to $\mathrm{T}$ cells in blood. It is therefore unclear whether patients with PCP are consistent with non-PCP patients with respect to their pulmonary T-cell subsets. Thus, defining the differences between circulating and pulmonary T-cell subsets in PCP patients and non-PCP patients is critical for better understanding the pathogenic immune mechanisms.

In this study, we characterized T-cell subset profiles (including Th1, Th2, Th9, and Th17 subpopulations, as well as corresponding $\mathrm{CD}^{+} \mathrm{T}$ cells) in the blood and lungs of HIV free, immunocompromised patients, according to their $P$. jirovecii status. Clinical correlations with T-cell populations were also evaluated.

\section{Materials and methods}

\section{Study subjects and sample collection}

We included a total of 44 immunocompromised patients in the Department of Respiratory and Critical Care Medicine at China-Japan Friendship Hospital between March 2017 and September 2018. All patients enrolled were defined as HIV-negative with one or more of the following immunosuppressive host conditions (anti-tumor chemotherapy for solid tumor or hematologic cancer, treatments for systemic disease, or immunosuppressive therapies) [26, 27]. The group was divided into two cohorts according to $P$. jirovecii status, which was determined via Giemsa and methenamine silver staining in bronchoalveolar lavage (BAL) fluid samples. Blood samples were collected and analyzed during the same period from 27 patients with PCP, and 17 patients without PCP. Concomitantly, pulmonary specimens were obtained during diagnostic procedures performed before patients initiated their therapeutic regimen. Due to the usage for clinical examination, BAL fluid samples were only obtained from 23 patients with PCP, and 14 patients without PCP. Clinical parameters for each patient were acquired through review of electronic medical records. Approval was obtained from the Ethics Committee of the China-Japan Friendship Hospital (No. 13018), and each patient provided informed consent.

\section{Sample processing}

Fiberoptic bronchoscopy with recovery of BAL fluid was performed according to a standardized protocol [28]. In brief, 5 aliquots of $40 \mathrm{~mL}$ saline solution were deposited into the lung lobe containing radiologic abnormalities. This solution was recollected, immediately centrifuged and placed on ice. Supernatants were discarded, and cells were resuspended in medium for flow cytometric analyses. Blood samples were prepared in heparin anticoagulant tubes by extracting peripheral blood mononuclear cells (PBMCs) isolated using Ficoll density gradient centrifugation (GE Healthcare, Sweden), and placed on the ice until analysis. Cell were washed, resuspended, and cultured in RPMI 1640 medium (Invitrogen, Carlsbad, CA, USA) containing 10\% Fetal Bovine Serum (Gibco, USA). 


\section{Multiparameter flow cytometric analysis}

We used intracellular cell staining (ICS) to detect intracellular cytokines as previously reported [29]. First, cells were stimulated by addition of phorbol myristate acetate (PMA; $50 \mathrm{ng} / \mathrm{mL}$, eBioscience, San Diego, California, USA) with ionomycin $(2 \mu \mathrm{g} / \mathrm{mL}$, eBioscience) in the presence of brefeldin A $(10 \mu \mathrm{g} / \mathrm{mL}$, eBioscience) for 4 hours at $37^{\circ} \mathrm{C}$. Cells were subsequently stained with extracellular anti-CD3-Percp-cy5.5, anti-CD4-APC-H7, anti-CD8-BV510 (all BD Biosciences, San Jose, California, USA) for $20 \mathrm{~min}$, followed by a fixation and permeabilization procedure using Perm/Wash Buffer (BD Biosciences). Thereafter, cells were washed with permeabilization buffer and incubated with intracellular antibodies including anti-IFN $\gamma$-Alexa 488, anti- IL4-BV711, anti-IL-9-PE, and anti-IL17-BV650 (all BD Biosciences). Appropriate isotypes were used. Finally, stained cells were acquired on an LSRII Fortessa cytometer (BD Biosciences). Data were analyzed using Flowjo software (Ashland, OR, USA).

\section{Statistical analysis}

Data management and analysis were performed using SPSS 16.0 software (Chicago, Illinois, USA). The Student's $t$-test or Mann-Whitney $U$ test were used to calculate differences in continuous variables between two groups. Categorical variables were compared using $\chi^{2}$ analysis or Fisher's exact test, as appropriate. Relationships between variables were identified using Pearson or Spearson correlation, as appropriate. Data are reported as mean \pm standard deviation (SD) or as median and interquartile range (IQR) $(25 \%, 75 \%)$. Significance was defined as having a $p$ value less than 0.05 .
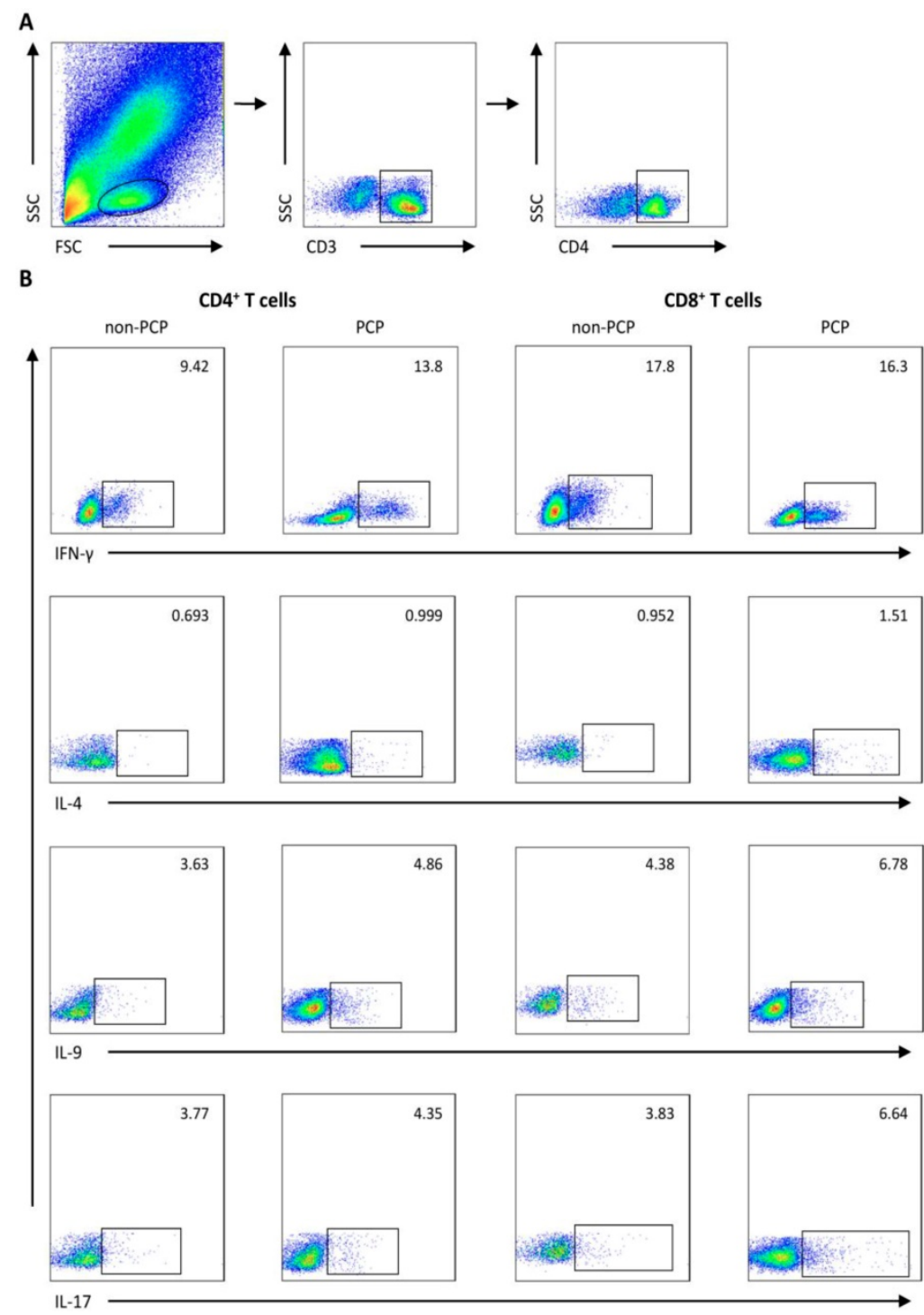

Figure 1. Representative flow cytometry data. Gating strategy and frequencies of T-cell subsets in blood from 1 ) a representative patient without Pneumocystis jirovecii pneumonia (PCP), and 2) a patient with PCP are shown. (A) Gating strategies of flow cytometric analysis. (B) Representative plots of subgroups of CD4+/CD8+ T cells sorted by IFN-y, IL-4, IL-9, and IL-17A expression. FSC: forward scatter characteristics; SSC: side scatter characteristics. 


\section{Results}

We used flow cytometry to quantify IFN-y, IL-4, IL-9 and IL-17-producing cells, which were defined as Th1/Tc1, Th2/Tc2, Th9/Tc9, Th17/Tc17 subsets of $\mathrm{CD}^{+}$and $\mathrm{CD}^{+} \mathrm{T}$ cells, respectively. Gating strategies for flow cytometry are depicted in Figure 1.

\section{Characteristics of study population}

Twenty-seven of the total of forty-four immunocompromised patients were diagnosed with PCP. The remaining 17 were $\mathrm{PCP}$ free. Table 1 presents the clinical characteristics of patients in each cohort. The mean age was $57.148 \pm 15.41$ years in the PCP cohort, and $61.94 \pm 13.069$ years in the cohort without $\mathrm{PCP}$, without significant difference. The sex ratios between $\mathrm{PCP}$ and non-PCP patients were also not different. In the patients with $\mathrm{PCP}$, underlying conditions were as follows. The most common underlying immunosuppressive diseases were interstitial lung disease $(48.15 \%)$, followed by systemic disease $(33.33 \%)$, hematological malignancy $(11.11 \%)$, solid tumor $(7.41 \%)$, and solid organ transplant $(7.41 \%)$. We observed no significant difference with regard to underlying diseases or the use of immunosuppressive agents between the two cohorts. Furthermore, patients in the PCP cohort were not more likely to receive prophylaxis at the time of the study than patients in the cohort without PCP.

Finally, white blood cells, lymphocyte counts, $\mathrm{CD} 4{ }^{+} \mathrm{T}$-cell counts, and CD8 ${ }^{+} \mathrm{T}$-cell counts were not different between cohorts with and without PCP.

Table 1. Summary of patient characteristics

\begin{tabular}{|c|c|c|c|}
\hline Characteristic & $\begin{array}{l}\text { non-PCP } \\
(\mathrm{n}=17)\end{array}$ & $\begin{array}{l}\text { PCP } \\
(\mathrm{n}=27)\end{array}$ & $\begin{array}{l}P \\
\text { value }\end{array}$ \\
\hline \multicolumn{4}{|l|}{ Demographics } \\
\hline Age (years), mean $\pm \mathrm{SD}$ & $61.94 \pm 13.07$ & $57.148 \pm 15.41$ & 0.294 \\
\hline Gender, male, n (\%) & $11(64.71)$ & $14(51.86)$ & 0.402 \\
\hline \multicolumn{4}{|l|}{ Underlying diseases, n (\%) } \\
\hline Solid tumor & $2(11.76)$ & $2(7.41)$ & 1.000 \\
\hline Hematological malignancy & $1(5.88)$ & $3(11.11)$ & 0.961 \\
\hline Solid organ transplantation & $1(5.88)$ & $2(7.41)$ & 1.000 \\
\hline Systemic disease & $8(47.06)$ & $9(33.33)$ & 0.363 \\
\hline Interstitial lung disease & $5(29.41)$ & $13(48.15)$ & 0.218 \\
\hline \multicolumn{4}{|l|}{$\begin{array}{l}\text { Immunosuppressive agents use, } \\
\mathrm{n}(\%)\end{array}$} \\
\hline Corticosteroids & $14(82.35)$ & $25(92.59)$ & 0.579 \\
\hline Anti-tumor chemotherapy & $1(5.88)$ & $4(14.81)$ & 0.674 \\
\hline T cell immunosuppressant ${ }^{\mathrm{a}}$ & $7(41.18)$ & $18(66.67)$ & 0.096 \\
\hline $\begin{array}{l}\text { Prophylaxis at the time of the } \\
\text { study }\end{array}$ & $4(23.53)$ & $3(11.11)$ & 0.501 \\
\hline \multicolumn{4}{|l|}{ Laboratory findings } \\
\hline $\begin{array}{l}\text { White blood cells }\left(10^{9} / \mathrm{L}\right) \text {, median } \\
\text { (IQR) }\end{array}$ & $7.05(6.08-11.48)$ & $\begin{array}{l}10.23 \\
(6.28-12.19)\end{array}$ & 0.286 \\
\hline Lymphocytes $\left(10^{9} / \mathrm{L}\right)$, median (IQR) & $0.36(0.21-0.73)$ & $0.54(0.35-1.01)$ & 0.339 \\
\hline $\mathrm{CD}^{+}$(cells $\left./ \mu \mathrm{L}\right)$, median (IQR) & $168(90-293)$ & $197(79.5-297.5)$ & 1.000 \\
\hline $\mathrm{CD}^{+}$(cells $\left./ \mu \mathrm{L}\right)$, median (IQR) & 154 (106-327) & $220(100-297.5)$ & 0.6038 \\
\hline
\end{tabular}

rituximab), and nucleoside analogues. Continuous variables are presented as median with IQR $(25 \%, 75 \%)$ or mean \pm SD. Other values are presented as numbers (\%). PCP, Pneumocystis jirovecii pneumonia; SD, standard deviation; IQR, interquartile range. $P<0.05$ was considered statistically significant.

\section{$\mathrm{CD4}^{+}$and $\mathrm{CD8}^{+} \mathrm{T}$ cells in the circulation and lungs of patients with PCP}

We initially determined $\mathrm{CD}^{+}$and $\mathrm{CD}^{+}{ }^{+} \mathrm{T}$-cell frequencies in peripheral blood and BAL fluid. The results, as shown in Figure 2, indicate that PCP did not caused significant increases or decreases in $\mathrm{CD}^{+}$ $\mathrm{T}$-cell percentages or $\mathrm{CD} 8^{+} \mathrm{T}$-cell percentages in blood relative to percentages in non-PCP $(P<0.0001)$, as were $\mathrm{CD}^{+} / \mathrm{CD}^{+}$T-cell ratios $(P<0.0001$, Figure $2 \mathrm{~A}-\mathrm{C})$. The T-cell frequencies in BAL fluid from PCP patients were also compared with those in non-PCP patients (Figure 2D-F); it is apparent that the fractionation parameters in the blood and BAL fluid of PCP patients were similar to those in the cohort without PCP.

\section{T-cell subsets in the circulation of patients with PCP}

The observation of similar frequencies of systemic and local $\mathrm{CD}^{+}, \mathrm{CD}^{+} \mathrm{T}$-cell from PCP and non-PCP groups prompted us to assess whether there also existed similar systemic frequencies in subsets of $\mathrm{CD}^{+}$and $\mathrm{CD} 8{ }^{+} \mathrm{T}$-cells between the two groups. As Figure 3 shows, no significant difference between the two groups was evident, as expected (Figure 3). In summary, we failed to differentiate immunocompromised patients with PCP from non-PCP patients in circulating T-cell subsets.

\section{Pulmonary expansion of Thl and TC9 in PCP cases}

Due to the similarity in circulating T-cell subset frequencies between patients with and without PCP, we asked whether pulmonary T-cell subsets are present in similar frequencies in the two groups. We therefore compared the T-cell patterns in the lungs of patient cohorts with and without PCP. The most striking result to emerge from this data is that the frequency of pulmonary Th1 cells in PCP patients was substantially increased, compared with those in patients without PCP $(P=0.0199$, Figure 4A). Interestingly, there was also an enhanced frequency of Tc9 cells in $\mathrm{PCP}$ patients, relative to patients without PCP $(P=0.0266$, Figure $4 \mathrm{G})$. Similar to results with peripheral T-cells, the T-cell subsets resident in the lungs did not differ between groups with and without PCP (Figure 4). Thus, $\mathrm{CD}^{+}$and $\mathrm{CD}^{+} \mathrm{T}$ cells in patients with $\mathrm{PCP}$ demonstrated local and selective enhanced frequencies of Th1 and Tc9 cells by comparison with non-PCP patients. 
A

$\mathrm{CD}^{+} \mathrm{T}$ cells in blood

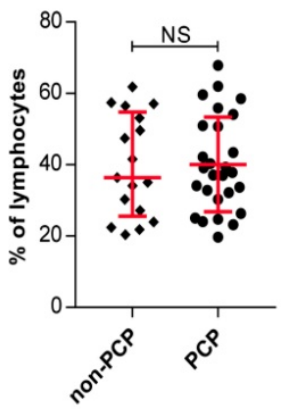

D

$\mathrm{CD4}^{+} \mathrm{T}$ cells in BAL fluid

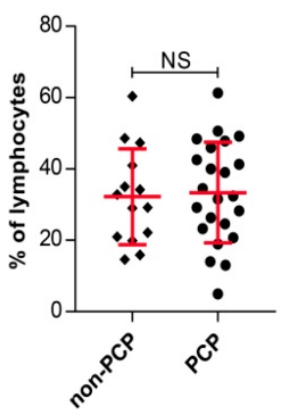

B

$\mathrm{CD}^{+} \mathrm{T}$ cells in blood

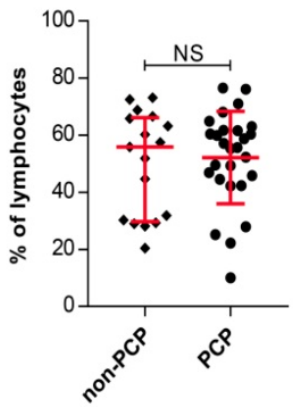

E $\mathrm{CD}^{+} \mathrm{T}$ cells in BAL fluid

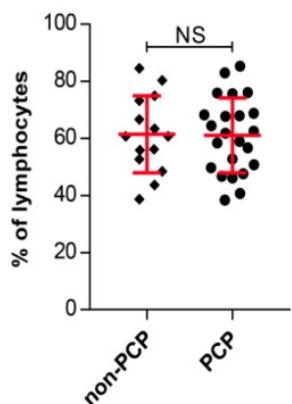

C

$\mathrm{CD4}^{+} / \mathrm{CD}^{+} \mathrm{T}$-cell ratio in blood

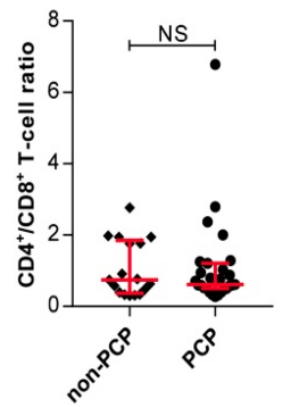

$\mathbf{F}$

$\mathrm{CD}^{+} / \mathrm{CD}^{+} \mathrm{T}$-cell ratio in $\mathrm{BAL}$ fluid

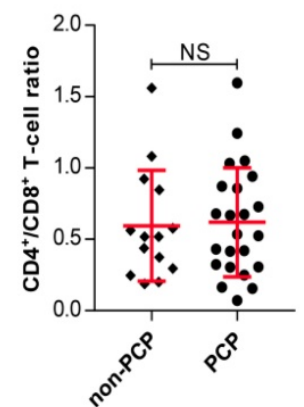

Figure 2. Frequencies of $C D 4^{+}$and $C D 8^{+} T$ cells, and $C D 4^{+} / C D 8^{+} T$-cell ratios. Percentages of $C D 4^{+} T$ cells $(\mathbf{A})$ and $C D 8^{+} T$ cells $(\mathbf{B})$, and $C D 4^{+} / C D 8^{+} T$-cell ratios $(\mathbf{C})$ in peripheral blood are exhibited. Frequencies of $C D 4^{+} T$ cells $(D)$ and $C D 8^{+} T$ cells $(E)$, and $C D 4^{+} / C D 8^{+} T$-cell ratios $(F)$ from bronchoalveolar lavage $(B A L)$ fluid are shown. For cells from blood, $n=27$ patients with Pneumocystis jirovecii pneumonia (PCP), and 17 patients without PCP. BAL fluid, $n=23$ in PCP, and 14 patients in non-PCP groups. ${ }^{* P}<0.05$; $* * P<0.01 ; * * * P<0.001$. NS, not significant.

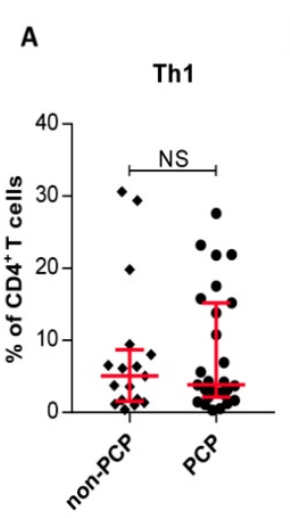

B

E

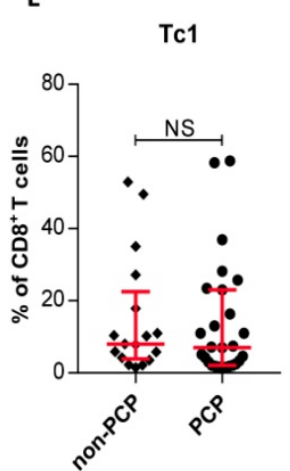

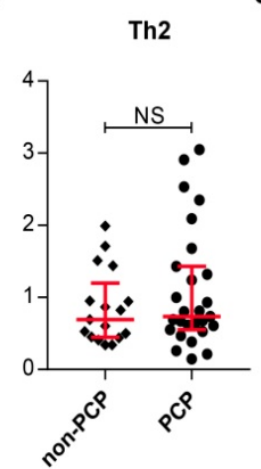

C

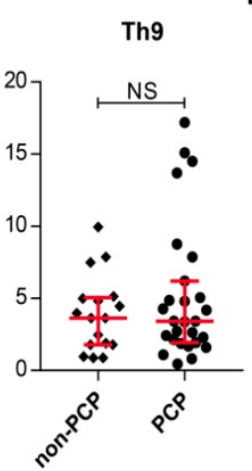

G<smiles>[Te]</smiles>

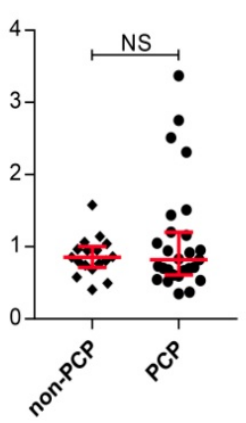

D

Th17

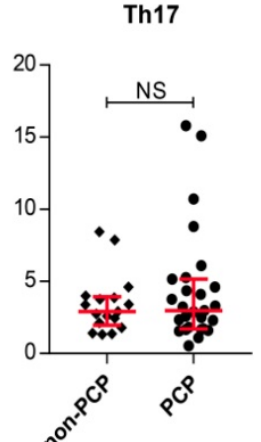

H

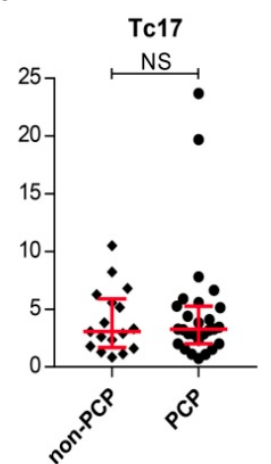

Figure 3. Intracellular cytokine expression in circulating CD4+ and CD8 ${ }^{+}$T cells. (A-H) Quantitative flow cytometric analyses of Th1/Tc1, Th2/Tc2, Th9/Tc9 and Th17/Tc17 subsets of $C D 4^{+} / C D 8^{+} T$ cells. Patients without Pneumocystis jirovecii pneumonia (PCP), $n=17$; patients with $P C P, n=27$. $* P<0.05 ; * * P<0.01$; $* * * P<0.001$. NS, not significant. 


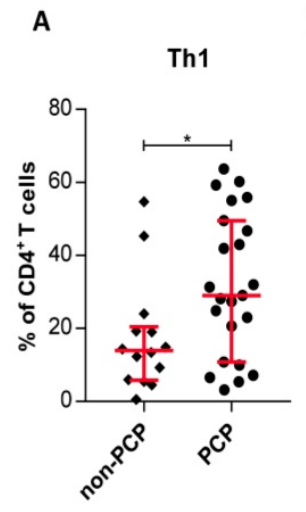

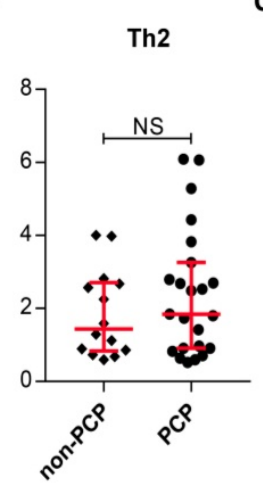

C

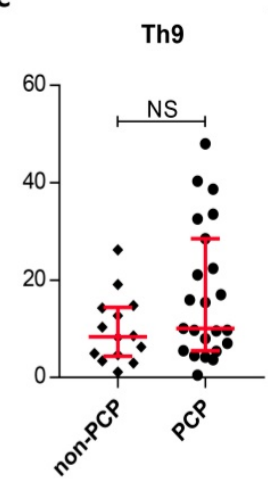

D

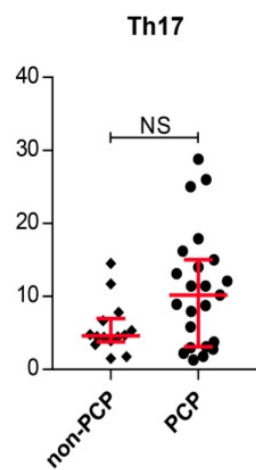

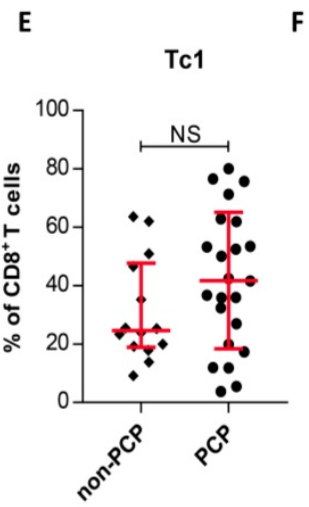

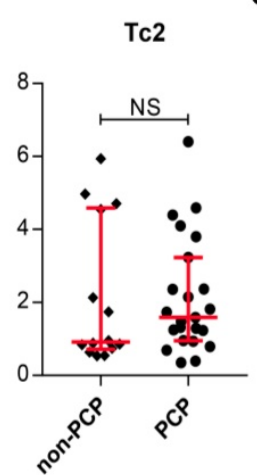

G

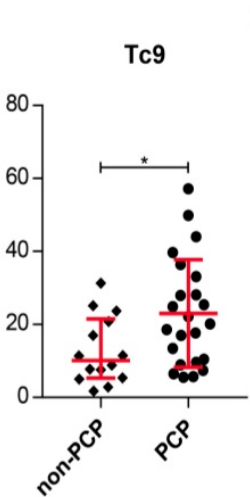

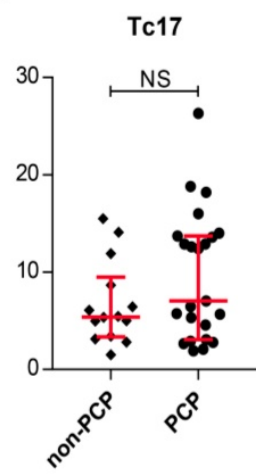

Figure 4. Differences in levels of pulmonary T-cell subsets. (A-H) Quantitation of IFN- ${ }^{+}, \mathrm{IL}-4^{+}$, IL-9+, and IL-17+ in the indicated T-cell subsets within the Pneumocystis jirovecii pneumonia $(P C P)$ cohort $(n=23)$ and non-PCP cohort $(n=14)$, as indicated. $* P<0.05 ; * * P<0.01 ; * * * P<0.001$. NS, not significant.

\section{Correlation between Th1 or Tc9 frequencies in the lungs and the circulating compartments, and disease severity in PCP patients}

Findings presented thus far suggest that Th1 and Tc9 subsets might mediate an immune response to $\mathrm{PCP}$, involving expansion relative to those in patients without PCP (Figure 4). To evaluate the relationship of these upregulated T-cell subsets in the lungs with PCP, we performed clinical correlation analysis with Th1 or Tc9 cells. There was no significant correlation between the blood and pulmonary percentages of Th1 cells (Figure 5A) and between the blood and pulmonary Tc9 frequencies (Figure $5 \mathrm{~B}$ ) in the $\mathrm{PCP}$ group. The results, as shown in Figure $5 \mathrm{C}$ and $5 \mathrm{D}$, indicate that the pulmonary Th1 cell levels in PCP patients exhibited an inverse correlation with the acute physiology and chronic health evaluation (APACHE) II score $(r=-0.463, P=0.0261)$ and sequential organ failure assessment (SOFA) score $(r=$ $-0.431, P=0.0401)$. Interestingly, the frequency of lung Tc9 cells in the PCP cohort positively correlated with the APACHE II score $(r=0.4605, P=0.0270$; Figure $5 \mathrm{E})$ and SOFA score $(r=0.4749, P=0.0220$; Figure 5F). Collectively, these results associated the host protective immune responses with the expansion of pulmonary Th1 cells and detrimental immune system activity with the expansion of pulmonary Tc9 subsets in patients with PCP.

\section{Discussion}

PCP is a challenging complication in immunocompromised patients due to the severity of symptoms and high mortality rates among those affected. Recently, Pneumocystis colonization has been found with increasing frequency in patients with chronic obstructive pulmonary disease, contributing to the impairment of pulmonary function [30, 31]. Although extensive research has been carried out on the role of $\mathrm{CD}^{+}{ }^{+} \mathrm{T}$ cells in mice, no single study exists which characterizes the response of either $\mathrm{CD}^{+}$or $\mathrm{CD}^{+} \mathrm{T}$-cell subsets in patients with PCP [32]. As reported, Struijk GH et al. have measured levels of Th1, Th2 and Th17 subsets in blood, in the PCP cohort of a group of renal transplant patients, and revealed no difference between them and the control cohort [25]. Recently, Th9 cells have been implicated in promoting pulmonary pathogenesis in a mouse model of Pneumocystis infection [21]. Therefore, on the basis of the present data underscoring the strong involvement of T-cells in response to $\mathrm{PCP}$, we present here an analysis of T-cell profiles in the circulation and in the lungs.

The present study provides a comprehensive 
A

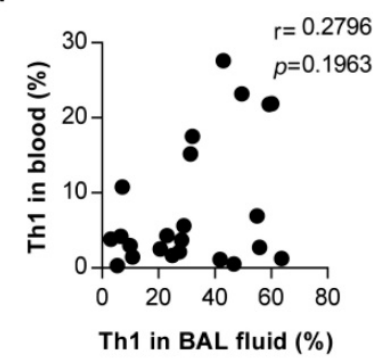

C

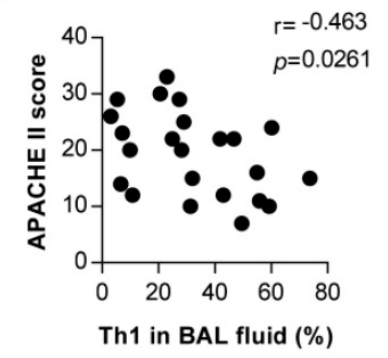

E

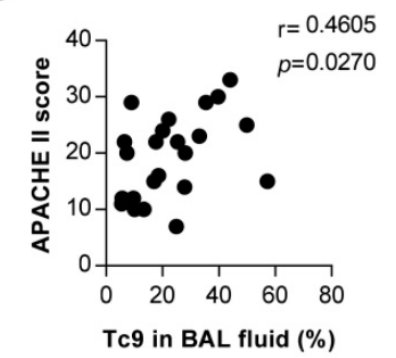

B

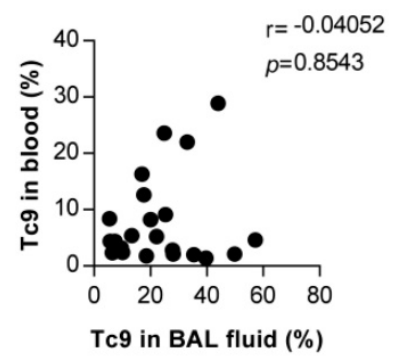

D

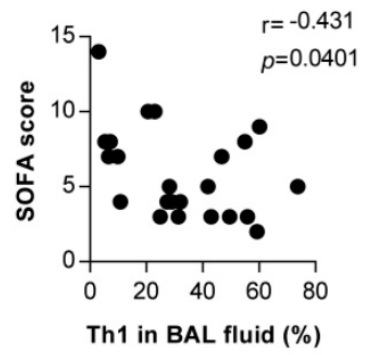

$\mathbf{F}$

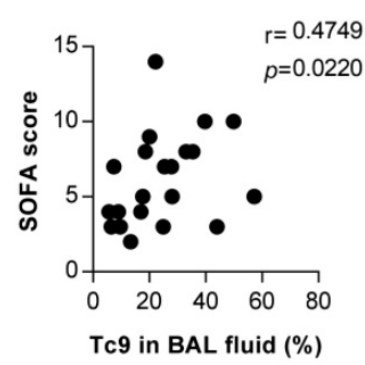

Figure 5. Correlation between frequencies of pulmonary Th1 or TC9 and blood compartments, and disease severity in Pneumocystis jirovecii pneumonia (PCP) patients. Correlation between frequencies of pulmonary and blood Th1 (A) and TC9 (B) in patients with PCP. Correlation between percentages of Th1 in bronchoalveolar lavage (BAL) fluid and the acute physiology and chronic health evaluation (APACHE) II score (C) and sequential organ failure assessment (SOFA) score (D). Correlation between percentages of pulmonary TC9 and APACHE II score and SOFA score was shown in (E) and (F), respectively. $n=23$. $P$ $<0.05$ was considered statistically significant.

assessment of the relative proportions of T-cell subsets in the circulation and in the lungs of immunocompromised patients with and without PCP, presenting an extensive characterization of T-cell patterns in local and systemic sites. Of note, we provide evidence here that blood T-cell subsets do not differentiate cohorts with and without PCP, but pulmonary Th1 and Tc9 appear to be significantly higher in patients with PCP than in those without. Thus, it might suggest that PCP blood phenotype did not parallel the T-cell activation pattern in the lungs. Our findings are thus in line with a previous study that reported no difference in Th1, Th2, and Th17 populations in the blood in patients with PCP following renal transplantation compared with control patients [25]. To our knowledge, our data represent the first investigation into immune activation of pulmonary T-cell subsets in patients with PCP. These findings provide better understanding of the pathogenesis of PCP, and provide a significant clinical basis for studying host adaptive immunity in non-HIV immunocompromised patients with PCP.

In our results, a similar reduction in $\mathrm{CD}^{+} \mathrm{T}$ cells and increased prevalence of $\mathrm{CD}^{+} \mathrm{T}$ cells occurs in blood and BAL fluid in the cohort with PCP relative to the cohort without PCP. This disagrees with the previously reported finding that the circulating $\mathrm{CD}^{+}{ }^{+}$-cell counts in PCP patients were significantly reduced compared to those in control patients [25]. We also failed to see a difference in circulating $\mathrm{T}$-cell subsets between patients with and without PCP, suggesting similar immunosuppression of peripheral T-cell immune activation. Although only a trend was observed toward higher T-cell mediated production of IL-4 in BAL fluid from PCP patients, its levels in PCP patient blood showed no significant differences than in control patients, suggesting it has distinct roles in circulation and in the lungs. It has recently been proposed that Th2 mediated immune responses are enhanced in P. murina-infected animals, where they play a beneficial role in immunity [33]. Further studies are needed to better characterize the role of Th2 in patients with PCP. Additionally, some studies have reported the significant roles of $\mathrm{CD} 4^{+} \mathrm{T}$-cell derived IL-22 and granulocyte-macrophage colony-stimulating factor in host defense against PCP [34-36]; however, we are missing important immune response in our present study. Thus, some studies should be conducted to investigate the significance of these cytokines.

Th17 cells have been seen to increase and possibly aid in clearance of Pneumocystis in animals [37]. Our findings revealed similar levels of circulating Th17 cells in patients with and without $\mathrm{PCP}$, in accord with recently reported data which saw no significant difference in Th17 percentages between cohorts with and without PCP in a study of renal transplant patients [25]. In spite of these findings, further studies are necessary to explore the role of Th17 in immunocompromised patients with and without PCP.

Our study revealed a significant increase in Th1 cells in BAL fluid in PCP patients in contrast to that of patients without PCP, suggesting involvement of these IFN- $\gamma$-producing $\mathrm{T}$ cells in the pulmonary inflammatory response, either in controlling or promoting. Previous studies have reported the size of IFN- $\gamma$-producing T cell populations in the lungs of $P$. murina infected animals [16]. Our findings appear to 
be in accord with this previous data. Several other studies have reported that IFN- $\gamma$-producing T cells are required for Pneumocystis-driven pulmonary inflammation. Paradoxically, Balb/c Stat4 $/$ - mice displayed susceptibility to $P$. murina infection in the lungs, while mice deficient in either IFN- $\gamma$ or IL-4 remain able to resolve infection [38, 39]. This discrepancy in experimental mice model studies might indicate the role of pulmonary Th1 cells is dispensable. Without consistence with these studies, we did find an adverse correlation between Th1 cell frequency and disease severity in the PCP patients, suggesting that pulmonary Th1 cells might play a protective role. By contrast to the percentages of IFN- $\gamma$-producing $\mathrm{T}$ cells in blood, we observed increased IFN- $\gamma$-producing $\mathrm{T}$ cells in BAL fluid in patients with PCP; thus, low circulating levels might be due to the increased migration into the lungs.

Recently, a great deal of attention has focused on the response of specific T- cell subsets, like Th1 [40], Th2 [18], Tregs [41, 42], and Tc [24] to PCP. In patients with $\mathrm{PCP}$, the host immune response appears to be mediated not only by $\mathrm{CD} 4^{+} \mathrm{T}$ cells, but also by $\mathrm{CD} 8^{+} \mathrm{T}$ cells, which may moderate $\mathrm{CD}^{+}$cell-mediated pathology by elevating Treg percentages in mice [14]. Several lines of evidence, suggest that the Tc1 subset of $\mathrm{CD}^{+}{ }^{+} \mathrm{T}$-cells compromises the host defense against Pneumocystis [23, 24]. Contrary to those results, our data revealed enrichment of Tc9 subsets in the BAL fluid of PCP patients relative to that of patients without PCP. The most obvious finding to emerge from this study is that the Tc9 percentage in BAL fluid positively correlated with the disease severity in patients with $\mathrm{PCP}$, implying that their contribution to immunopathogenesis is detrimental to PCP patients. Although we did not find a significant correlation between Tc1 prevalence and disease severity in the PCP cohort, these data corroborate the conclusions of previous studies, which suggest that $\mathrm{Tc} 1 \mathrm{CD} 8{ }^{+} \mathrm{T}$ cells are not a primary contributor to lung damage [24]. Indeed, the PCP patient specific expanded pulmonary Tc9 cell population could be targeted in the future to treat PCP in human patients.

IL-9 is reported to have a defect in patients with chronic mucocutaneous candidiasis [43], but its role in PCP remains unclearly defined. Increased IL-9 levels and Th9 cells have recently been seen in $P$. murina-infected mice, where their inverse correlation with clearance of $P$. murina suggested a role in pathogenesis [21]. However, no studies have investigated the role(s) of Th9/Tc9 in the circulation or lungs of patients with PCP. In this context, our results suggest that a Tc9-related rather than a Th9-related response, is likely to perform a significant detrimental function in PCP cohorts. Together, these observations suggest that Th9 cell expansion plays an immunopathogenic role in humans as is observed in mice, and provide insight into the role of Tc9 cells in PCP.

Our observations presented here are intriguing in the circulating and pulmonary T-cell immune responses in HIV-negative patients with PCP. Furthermore, in our study, only pulmonary Th1 and TC9 could distinguish patients with and without PCP. We did not find any correlation between the pulmonary Th1 or Tc9 frequencies and the circulating compartments in patients with PCP. These results are supported by earlier studies in which blood T-cell populations did not correlate with those in BAL fluid of HIV-negative patients with PCP [27]. However, our data contrasts with data from a study of HIV-positive patients with PCP, where a correlation was found between T-cell populations in alveoli and blood [27, 44]. Thus, our findings suggest that local $\mathrm{T}$ cells in the lungs might not reflect systemic events in the circulations. Mitigating the value of this discovery, acquisition of BAL fluid samples is a relatively invasive procedure to perform for diagnostic purposes, thus less invasive methods or predictive factors are needed.

We acknowledge some possible weaknesses are present in our study. With a small sample size and single study center, caution must be applied, as the findings might not be accurately representative. Another note of caution is due here since we are limited to subjects that were admitted to the ICU. Since our study was limited to Giemsa and methenamine silver staining of Pneumocystis using BAL fluid samples, which were known to have low sensitivity for detection of Pneumocystis and have the potential to be positive in patients with colonization, it was possible to misclassify some patients. Additional studies will be warranted to dissect the response mechanisms of specific subsets of $\mathrm{T}$ cells thoroughly. Further studies, which take these factors into account, will need to be undertaken.

\section{Conclusions}

In summary, the data in this study complements data from animal studies and expands the available human data, and indicates that local expansion of Th1 and Tc9 subsets may play protective and detrimental roles in PCP, respectively. More broadly, the combination of findings presented here are assumed to have relevant implications for better understanding of T-cell responses, which may support development of immunotherapies aimed at specific subsets of Th1 or Tc9 cells for Pneumocystis infection. 


\section{Abbreviations}

HIV: human immunodeficiency virus; PCP: Pneumocystis jirovecii pneumonia; ICU: intensive care units (ICU); HC: healthy controls; BAL: bronchoalveolar lavage; PBMCs: peripheral blood mononuclear cells; ICS: intracellular cell staining; PMA: phorbol myristate acetate; SD: standard deviation; IQR: interquartile range; APACHE: acute physiology and chronic health evaluation; SOFA: sequential organ failure assessment.

\section{Acknowledgements}

All authors thank the staff of the Department of Respiratory and Critical Care Medicine at China-Japan Friendship Hospital for their assistance in screening study subjects and collecting samples throughout the study. We would like to gratefully acknowledge the patients and healthy donors for their involvement.

\section{Funding}

This work was supported by grants from the National Natural Science Foundation of China (grant numbers 1470270 and 81870072); the National Key Research and Development Program of China (grant number 2016YFC1304300); the Capital Clinical Features Applied Research and Achievement Promotion Project of Beijing, China (grant number Z161100000516116); the Major projects of the national natural science foundation of China (grant number 81490534); and the Fundamental Research Funds for the Central Universities, China (grant number 3332018183).

\section{Ethics approval}

Approval was obtained from the Ethics Committee of the China-Japan Friendship Hospital (No. 13018), and each patient provided informed consent.

\section{Competing Interests}

The authors have declared that no competing interest exists.

\section{References}

1. Maini R, Henderson KL, Sheridan EA, et al. Increasing Pneumocystis pneumonia, England, UK, 2000-2010. Emerg Infect Dis. 2013; 19: 386-92.

2. Thomas CF, Jr., Limper AH. Pneumocystis pneumonia. N Engl J Med. 2004; 350: 2487-98.

3. Reid AB, Chen SC, Worth LJ. Pneumocystis jirovecii pneumonia in non-HIV-infected patients: new risks and diagnostic tools. Curr Opin Infect Dis. 2011; 24: 534-44

4. Schmidt JJ, Lueck C, Ziesing S, et al. Clinical course, treatment and outcome of Pneumocystis pneumonia in immunocompromised adults: a retrospective analysis over 17 years. Crit Care. 2018; 22: 307.

5. Morris A, Norris KA. Colonization by Pneumocystis jirovecii and its role in disease. Clin Microbiol Rev. 2012; 25: 297-317.

6. Monnet X, Vidal-Petiot E, Osman D, et al. Critical care management and outcome of severe Pneumocystis pneumonia in patients with and without HIV infection. Crit Care. 2008; 12: R28.
7. Festic E, Gajic $\mathrm{O}$, Limper $\mathrm{AH}$, et al. Acute respiratory failure due to pneumocystis pneumonia in patients without human immunodeficiency virus infection: outcome and associated features. Chest. 2005; 128: 573-9.

8. Fillatre $\mathrm{P}$, Decaux $\mathrm{O}$, Jouneau $\mathrm{S}$, et al. Incidence of Pneumocystis jiroveci pneumonia among groups at risk in HIV-negative patients. Am J Med. 2014; 127: 1242 e11-7.

9. Kelly MN, Shellito JE. Current understanding of Pneumocystis immunology. Future Microbiol. 2010; 5: 43-65.

10. Hoving JC, Kolls JK. New advances in understanding the host immune response to Pneumocystis. Curr Opin Microbiol. 2017; 40: 65-71.

11. Harmsen AG, Stankiewicz M. Requirement for CD4+ cells in resistance to Pneumocystis carinii pneumonia in mice. J Exp Med. 1990; 172: 937-45.

12. Mansharamani NG, Balachandran D, Vernovsky I, et al. Peripheral Blood CD4 + T-Lymphocyte Counts During Pneumocystis carinii Pneumonia in Immunocompromised Patients Without HIV Infection. Chest. 2000; 118: 712-20.

13. Roths JB, Sidman CL. Both Immunity and Hyperresponsiveness to Pneumocystis carinhi Result from Transfer of CD4+ but not CD8+ T Cells into Severe Combined Immunodeficiency Mice. J Clin Invest. 1992; 90: 673-8.

14. Swain SD, Meissner NN, Harmsen AG. CD8 T cells modulate CD4 T-cell and eosinophil-mediated pulmonary pathology in pneumocystis pneumonia in B-cell-deficient mice. Am J Pathol. 2006; 168: 466-75.

15. Bhagwat SP, Gigliotti F, Xu H, et al. Contribution of $\mathrm{T}$ cell subsets to the pathophysiology of Pneumocystis-related immunorestitution disease. Am J Physiol Lung Cell Mol Physiol. 2006; 291: L1256-66.

16. Shellito JE, Tate C, Ruan S, et al. Murine CD4+ T lymphocyte subsets and host defense against Pneumocystis carinii. J Infect Dis. 2000; 181: 2011-7.

17. Garvy BA, Wiley JA, Gigliotti F, et al. Protection against Pneumocystis carinii pneumonia by antibodies generated from either $\mathrm{T}$ helper 1 or $\mathrm{T}$ helper 2 responses. Infect Immun. 1997; 65: 5052-6.

18. Myers RC, Dunaway CW, Nelson MP, et al. STAT4-dependent and -independent Th2 responses correlate with protective immunity against lung infection with Pneumocystis murina. J Immunol. 2013; 190: 6287-94.

19. Rudner XL, Happel KI, Young EA, et al. Interleukin-23 (IL-23)-IL-17 cytokine axis in murine Pneumocystis carinii infection. Infect Immun. 2007; 75: 3055-61.

20. Zelante T, De Luca A, Bonifazi P, et al. IL-23 and the Th17 pathway promote inflammation and impair antifungal immune resistance. Eur J Immunol. 2007; 37: 2695-706.

21. Li T, Rong HM, Zhang C, et al. IL-9 Deficiency Promotes Pulmonary Th17 Response in Murine Model of Pneumocystis Infection. Front Immunol. 2018; 9: 1118

22. Wright TW, Gigliotti F, Finkelstein JN, et al. Immune-mediated inflammation directly impairs pulmonary function, contributing to the pathogenesis of Pneumocystis carinii pneumonia. J Clin Invest. 1999; 104: 1307-17.

23. McAllister F, Ruan S, Steele C, et al. CXCR3 and IFN protein-10 in Pneumocystis pneumonia. J Immunol. 2006; 177: 1846-54.

24. McAllister F, Steele C, Zheng M, et al. T cytotoxic-1 CD8+ T cells are effector cells against pneumocystis in mice. J Immunol. 2004; 172: 1132-8.

25. Struijk GH, Gijsen AF, Yong SL, et al. Risk of Pneumocystis jiroveci pneumonia in patients long after renal transplantation. Nephrol Dial Transplant. 2011; 26: 3391-8.

26. De Pauw B, Walsh TJ, Donnelly JP, et al. Revised definitions of invasive fungal disease from the European Organization for Research and Treatment of Cancer/Invasive Fungal Infections Cooperative Group and the National Institute of Allergy and Infectious Diseases Mycoses Study Group (EORTC/MSG) Consensus Group. Clin Infect Dis. 2008; 46: 1813-21.

27. Iriart $\mathrm{X}$, Witkowski $\mathrm{B}$, Cassaing $\mathrm{S}$, et al. Alveolar and blood $\mathrm{T}$ lymphocyte profiles in Pneumocystis jirovecii-positive patients: effects of HIV status. J Infect Dis. 2011; 204: 544-53.

28. Muhlethaler K, Bogli-Stuber K, Wasmer S, et al. Quantitative PCR to diagnose Pneumocystis pneumonia in immunocompromised non-HIV patients. Eur Respir J. 2012 : 39. 971-8.

29. Hertoghs KM, Moerland PD, van Stijn A, et al. Molecular profiling of cytomegalovirus-induced human CD8+ T cell differentiation. J Clin Invest. 2010; 120: 4077-90.

30. Morris A, Sciurba FC, Lebedeva IP, et al. Association of chronic obstructive pulmonary disease severity and Pneumocystis colonization. Am J Respir Crit Care Med. 2004; 170: 408-13.

31. Calderon EJ, Rivero L, Respaldiza N, et al. Systemic inflammation in patients with chronic obstructive pulmonary disease who are colonized with Pneumocystis jiroveci. Clin Infect Dis. 2007; 45: e17-9.

32. Phair J, Munoz A, Detels R, et al. The risk of Pneumocystis carinii pneumonia among men infected with human immunodeficiency virus type 1 . Multicenter AIDS Cohort Study Group. N Engl J Med. 1990; 322: 161-5.

33. Eddens T, Elsegeiny W, Nelson MP, et al. Eosinophils Contribute to Early Clearance of Pneumocystis murina Infection. J Immunol. 2015; 195: 185-93.

34. McAllister F, Steele C, Zheng M, et al. In vitro effector activity of Pneumocystis murina-specific T-cytotoxic-1 CD8+ T cells: role of granulocyte-macrophage colony-stimulating factor. Infect Immun. 2005; 73: 7450-7.

35. Carmona EM, Kottom TJ, Hebrink DM, et al. Glycosphingolipids mediate pneumocystis cell wall beta-glucan activation of the IL-23/IL-17 axis in human dendritic cells. Am J Respir Cell Mol Biol. 2012; 47: 50-9.

36. Paine R, 3rd, Preston AM, Wilcoxen S, et al. Granulocyte-macrophage colony-stimulating factor in the innate immune response to Pneumocystis carinii pneumonia in mice. J Immunol. 2000; 164: 2602-9. 
37. Perez-Nazario N, Rangel-Moreno J, O'Reilly MA, et al. Selective ablation of lung epithelial IKK2 impairs pulmonary Th17 responses and delays the clearance of Pneumocystis. J Immunol. 2013; 191: 4720-30.

38. Garvy BA, Ezekowitz RA, Harmsen AG. Role of gamma interferon in the host immune and inflammatory responses to Pneumocystis carinii infection. Infect Immun. 1997; 65: 373-9.

39. Garvy BA, Gigliotti F, Harmsen AG. Neutralization of interferon-gamma exacerbates pneumocystis-driven interstitial pneumonitis after bone marrow transplantation in mice. J Clin Invest. 1997; 99: 1637-44.

40. Meissner N, Swain S, McInnerney K, et al. Type-I IFN signaling suppresses an excessive IFN-gamma response and thus prevents lung damage and chronic inflammation during Pneumocystis (PC) clearance in CD4 T cell-competent mice. Am J Pathol. 2010; 176: 2806-18.

41. McKinley L, Logar AJ, McAllister F, et al. Regulatory $\mathrm{T}$ cells dampen pulmonary inflammation and lung injury in an animal model of pneumocystis pneumonia. J Immunol. 2006; 177: 6215-26.

42. Hori S, Carvalho TL, Demengeot J. CD25+CD4+ regulatory T cells suppress $\mathrm{CD} 4+\mathrm{T}$ cell-mediated pulmonary hyperinflammation driven by Pneumocystis carinii in immunodeficient mice. Eur J Immunol. 2002; 32: 1282-91.

43. Becker KL, Rosler B, Wang X, et al. Th2 and Th9 responses in patients with chronic mucocutaneous candidiasis and hyper-IgE syndrome. Clin Exp Allergy. 2016; 46: 1564-74.

44. Iriart X, Witkowski B, Courtais C, et al. Cellular and cytokine changes in the alveolar environment among immunocompromised patients during Pneumocystis jirovecii infection. Med Mycol. 2010; 48: 1075-87. 